livraisons

d'Histoire

de l'Architecture

\section{Livraisons de l'histoire de l'architecture}

15 | 2008

Chambres de commerces

\title{
Un palais néo-régionaliste pour une grande institution : la construction de la nouvelle bourse de commerce de Lille par Louis-Marie Cordonnier (1906-1920)
}

A Neo-Regionalist palace for a great institution. The erection of the new Lille Stock Exchange by Louis-Marie Cordonnier (1906-1920)

Ein neoregionalistischer Palast für eine große Institution. Die neue Handelsbörse von Lille, Bau des Architekten Louis-Marie Cordonnier (1906-1920)

\section{Olivier Liardet}

\section{OpenEdition}

Journals

Édition électronique

URL : http://journals.openedition.org/lha/168

DOI : 10.4000/lha. 168

ISSN : $1960-5994$

Éditeur

Association Livraisons d'histoire de l'architecture - LHA

Édition imprimée

Date de publication : 10 juin 2008

Pagination : 67-88

ISSN : 1627-4970

Référence électronique

Olivier Liardet, « Un palais néo-régionaliste pour une grande institution : la construction de la nouvelle bourse de commerce de Lille par Louis-Marie Cordonnier (1906-1920) », Livraisons de l'histoire de l'architecture [En ligne], 15 | 2008, mis en ligne le 10 juin 2010, consulté le 19 avril 2019. URL : http:// journals.openedition.org//ha/168; DOI : 10.4000/lha.168

Ce document a été généré automatiquement le 19 avril 2019

Tous droits réservés à l'Association LHA 


\title{
Un palais néo-régionaliste pour une grande institution : la construction de la nouvelle bourse de commerce de Lille par Louis-Marie Cordonnier (1906-1920)
}

\author{
A Neo-Regionalist palace for a great institution. The erection of the new Lille \\ Stock Exchange by Louis-Marie Cordonnier (1906-1920)
}

Ein neoregionalistischer Palast für eine große Institution. Die neue Handelsbörse von Lille, Bau des Architekten Louis-Marie Cordonnier (1906-1920)

\section{Olivier Liardet}

Si le nom de l'architecte Louis-Marie Cordonnier (1854-1940) demeure indissociablement lié à Lille et au Nord de la France, sa région natale, sa renommée contemporaine a largement dépassé les frontières nationales grâce à ses projets lauréats pour le palais de la bourse d'Amsterdam (1884, réalisé par Hendrik Berlage), la façade de la cathédrale de Milan (1887) ou encore le palais de la Paix de La Haye voulu par Andrew Carnegie (1905-1913). Bien qu'il eût été dépourvu des lauriers du grand prix de Rome, son élection à l'Institut en 1911, de même que sa dernière œuvre, la basilique Sainte-Thérèse de Lisieux, achevée par son fils, viennent consacrer l'un des architectes français les plus célèbres du début $\mathrm{du} \mathrm{XX}^{\mathrm{e}}$ siècle. Pour autant, la carrière et l'œuvre de Cordonnier demeurent encore peu connus et ce constat relève du paradoxe si l'on tient compte qu'à la différence de nombre de ses confrères, son fonds d'agence couvrant une large part de son activité est aujourd'hui en grande partie conservé dans les archives publiques ${ }^{1}$. Ainsi, à l'exception notable de ses hôtels de ville, qui ont fait l'objet d'une étude ces dernières années et dans une moindre mesure de l'Opéra de Lille, l'ensemble de ses travaux reste à redécouvrir et cette remarque s'applique en particulier à son édifice lillois le plus emblématique, point d'orgue de toutes les spéculations historiques et urbaines: la nouvelle Bourse de Commerce qui, sous sa veste néo-régionaliste, marque l'entrée de la 
capitale du Nord dans la modernité et souligne son rôle dans l'économie nationale comme dans le renouveau des zones dévastées par les combats de la Grande Guerre.

\section{L'institution consulaire}

2 La première manifestation d'une représentation commerciale auprès de l'État remonte à Henri IV qui crée le 13 avril 1601 une commission de seize membres pour étudier les moyens de nature à restaurer le commerce du royaume. Cette représentation perdure par intermittence jusqu'en 1661, date de création d'un conseil du commerce refondé par arrêt du Conseil d'État du 29 juin 1700. L'importante réforme de 1722 transforme le Conseil en bureau du commerce qui perdure jusqu'à sa suppression le 27 septembre 1791. Cependant, la représentation commerciale auprès de certaines municipalités existait antérieurement à celle placée auprès du gouvernement. Si la plus ancienne chambre de commerce est celle de Marseille créée le 5 août 1599, la première création flamande, Dunkerque, ne date que de février 1700. Au regard des services essentiels rendus au commerce par les institutions consulaires, le gouvernement royal décide la création de chambres de commerce dans un certain nombre de villes dont Lille, par arrêt du Conseil d'État du 30 août 1701, afin d'informer le Conseil du commerce de tous les besoins généraux du commerce de leur ville et de la région qu'elles représentaient. La création de la Chambre de Lille n'intervient toutefois que le 31 juillet 1714 par arrêt du Conseil d'État avec installation effective le $1^{\mathrm{er}}$ avril $1715^{2}$. Elle est la conséquence du retour de la Flandre française et de sa capitale au royaume de France après la Paix d'Utrecht (1713) marquant la fin de la Guerre de Succession d'Espagne et de l'occupation hollandaise. À la Révolution, les chambres de commerce sont supprimées en même temps que le bureau du commerce, mais la nécessité de leur action amène leur rétablissement le 3 nivôse an XI (24 décembre 1802) dans vingt-deux villes dont Dunkerque et Lille, sous l'autorité d'un conseil général du commerce établi auprès du ministre de l'Intérieur. Celle de Lille est installée le 14 février 1803 avec comme circonscription les arrondissements de Lille, Douai, Cambrai, Valenciennes et Avesnes-sur-Helpe. Son étendue ne cesse de se réduire au cours du XIX ${ }^{e}$ siècle par la création d'autres chambres: Valenciennes (1836), Roubaix et Tourcoing (1872), Douai (1872), Cambrai (1880), Armentières et Avesnes-sur-Helpe (1887). À partir de 1887, la Chambre de Lille ne couvre plus que huit cantons lillois. Enfin en 1966, les Chambres de Lille, Roubaix et Tourcoing décident de fusionner afin de donner une plus grande efficacité à leur action. La grande réforme du 9 avril 1898 marque un tournant dans le rôle des chambres de commerce qui sont déclarées établissement publics avec extension de leurs attributions et la possibilité de contracter des emprunts pour concourir aux dépenses de construction de bourses ou de palais consulaires et de participer aux frais d'exploitation des établissements qu'elles sont autorisées à fonder tels qu'écoles et musées commerciaux, magasins, entrepôts, etc. L'emploi du produit de centimes additionnels à la patente leur permet de gager les emprunts et de couvrir ces dépenses.

\section{La Vieille Bourse}

3 La construction de la Vieille Bourse de Lille était destinée à donner un abri aux marchands qui se réunissaient auparavant autour de la fontaine au change ${ }^{3}$. Autorisés par lettres patentes de Philippe IV, roi d'Espagne, en date du 7 juin 1651, les travaux se 
déroulent en 1662-1663. Le bâtiment se compose d'une cour intérieure bordée de galeries servant aux marchands, le tout étant entouré de maisons particulières au nombre de vingt-quatre dont les terrains avaient été vendus par le Magistrat qui ne s'était réservé que le sol de la cour avec les quatre entrées, les tourelles du toit et les balcons des deux entrées principales. Les propriétaires des maisons devaient se conformer au dessin donné par Julien Destrez, fabricant de meubles, maître-arpenteur, maître des œuvres de la ville de 1642 à 1677 et construire les maisons à leur frais. Les nombreuses études réalisées sur l'édifice depuis le $\mathrm{XIX}^{\mathrm{e}}$ siècle ont montré la place majeure de cet édifice dans l'architecture lilloise et flamande, en particulier les implications urbanistiques de l'édifice sur l'espace de la ville. Relevons simplement que la Vieille Bourse de Lille constitue à la fois un exemple-type de l'architecture maniériste septentrionale à la décoration foisonnante d'inspiration flamande et un modèle à suivre, bientôt contrecarré par l'annexion française et l'acclimatation d'une architecture plus sobre, empreinte de rigueur et soucieuse de composition plus que de décoration. La dégradation de l'édifice semble avoir commencé dès le XVIII ${ }^{e}$ siècle et la Vieille Bourse devint rapidement trop exiguë pour recevoir les nombreux négociants et courtiers venant y traiter leurs affaires. C'est ainsi que ces derniers prirent l'habitude de se réunir dans les cafés situés autour de la Grand'Place.

\section{Une Chambre itinérante au $\mathrm{XIX}^{\mathrm{e}}$ siècle : vers un bâtiment consulaire spécifique}

4 En 1802, la Chambre rentre en possession de l'ancienne Bourse de Lille dite Vieille Bourse et obtient l'évacuation du local par les boutiques indûment installées. Peu après, elle fait démolir la partie supérieure du campanile devenue trop vétuste. Dès 1809, la Chambre envisage des travaux de rénovation qui ne seront pas toujours suivis d'exécution ${ }^{4}$. En 1812, les façades sont partiellement restaurées et deux portes en fer exécutées. La destruction de l'ensemble du campanile est envisagée en 1828 sur la demande de deux propriétaires dont les locaux se trouvaient sous celui-ci : acceptée par l'architecte de la ville, elle est en définitive refusée par la municipalité. À partir de l'ordonnance royale du 16 juin 1832, lorsqu'une chambre de commerce et une Bourse existent dans la même ville, la Bourse devient la propriété de la Chambre, l'entretien et la conservation du bâtiment étant alors partagés avec la municipalité. Pendant tout le XIX siècle, la Chambre et la Ville font ainsi réaliser des travaux de restauration et commandent des projets de réutilisation de l'ancien édifice afin de lui rendre son lustre et de l'adapter aux nouvelles tâches consulaires. En 1842, l'architecte de la ville, Charles Benvignat (1805-1877), rétablit deux des portes de la Vieille Bourse ${ }^{5}$. En 1844, les enseignes sont rabaissées au-dessous du niveau du premier étage et Benvignat dresse un devis pour la reconstitution du campanile sur des fonds municipaux. Les travaux sont achevés en 1845 par l'entrepreneur Frémÿ. Les façades sont restaurées à la même époque et les sculptures sont rétablies dans un état originel par la création ou le remodelage d'éléments décoratifs (guirlandes, cartouches, etc) $)^{6}$.

5 À l'avènement du Second Empire, la Chambre prend la décision d'élever une statue de Napoléon $\mathrm{I}^{\mathrm{er}}$ en costume de sacre afin de rappeler l'importance des décisions prises par l'empereur en faveur du développement des industries lainière et sucrière si importantes dans la région. Le monument est exécuté en bronze par Henri Lemaire (1798-1880) et placé sur un piédestal dessiné par Benvignat. L'inauguration eut lieu le 3 décembre $1854^{7}$. 
Dès 1853, les membres de la Chambre décident de constituer un panthéon dans l'idée d'honorer les figures qui ont contribué à la richesse et à la prospérité de l'industrie nationale. Cette décoration prend place dans les galeries intérieures sous la forme de panneaux décorés en pierre blanche soutenant de grandes plaques de marbre noir portant des inscriptions (actes rappelant les institutions les plus importantes pour le commerce et l'industrie et les œuvres des grands savants et inventeurs dont les travaux ont permis le développement de l'industrie et du commerce) surmontés des bustes en bronze et des emblèmes allégoriques correspondants ${ }^{8}$. En 1860, les façades extérieures et intérieures sont restaurées avec soin. Quatre ans plus tard, la Chambre émet l'idée de couvrir la cour de la Vieille Bourse, mais les difficultés résultant de la situation de l'immeuble dévolu à vingt-quatre propriétaires font abandonner le projet qui est repris en 1882 et de nouveau abandonné pour raisons financières. Depuis sa création, la Chambre de Lille siège dans une salle de l'Hôtel de Ville . Elle y demeure jusqu'en août $1868^{10}$, puis s'installe jusqu'au $1^{\mathrm{er}}$ octobre 1898 dans un local situé au-dessus de la salle des Pas-Perdus de la nouvelle gare.

6 En 1894, la Chambre charge une commission de trouver un immeuble destiné à réunir ses services et le musée commercial. C'est l'occasion pour les membres de l'assemblée consulaire de faire valoir leur vision de l'institution: «M. Darnel [Léonard Darnel, imprimeur et membre de la Chambre] est d'avis qu'il conviendrait que la chambre de commerce ait une situation plus indépendante et qu'on devrait dès maintenant se préoccuper d'un nouveau local. Il propose en conséquence de nommer une commission pour étudier les voies et moyens $»^{11}$. Une commission spéciale est nommée peu après afin de réfléchir à la question d'une nouvelle installation ${ }^{12}$. Plusieurs solutions sont alors envisagées notamment l'occupation de l'ancien Mont-de-Piété dévolu au musée commercial, rue du Lombard, et surtout l'expropriation complète de la Vieille Bourse, rétablie dans un état primitif par la suppression des magasins, le couvrement de la cour et l'installation des bureaux commerciaux au rez-de-chaussée et au premier étage tandis que la Chambre et le musée occuperaient l'étage supérieur ${ }^{13}$. Le projet proposé par l'adjoint au maire, Émile Gavelle ${ }^{14}$, aurait dû être supporté financièrement par la ville moyennant un loyer sur quarante années de la Chambre, et fut abandonné en raison de l'importance de la dépense ${ }^{15}$. En 1904, l'idée d'un transfert dans le marché Saint-Nicolas situé derrière la Grand-Garde est évoquée et rapidement adoptée. Cet emplacement était un des plus intéressants puisqu'il permettait un vis-à-vis avec la Vieille Bourse. Le projet suspendu puis repris fut définitivement abandonné en octobre $1905^{16}$ car il aurait entraîné la démolition de la façade de la Grand-Garde (construite en 1717) à laquelle se refusait la Ville en raison de son caractère historique.

\section{Une visibilité urbaine exceptionnelle}

7 À partir de 1904, la nouvelle municipalité, dirigée par l'industriel du textile Charles Delesalle, s'engage dans une active politique de redressement financier et de grands travaux d'urbanisme ${ }^{17}$. En 1905, la Ville vote l'ouverture d'un boulevard reliant Lille à Roubaix et Tourcoing, accessible à l'automobile et au tramway dont le départ se situe sur la place du Théâtre, édifice construit par Michel Lequeux à la fin du XVIII ${ }^{e}$ siècle et détruit par un incendie en avril 1903. Les travaux d'urbanisme s'achèvent en 1908 (ill. 1). 
III. 1 : Émile Dubuisson, plan des projets d'aménagement du quartier du théâtre avec l'arrivé du

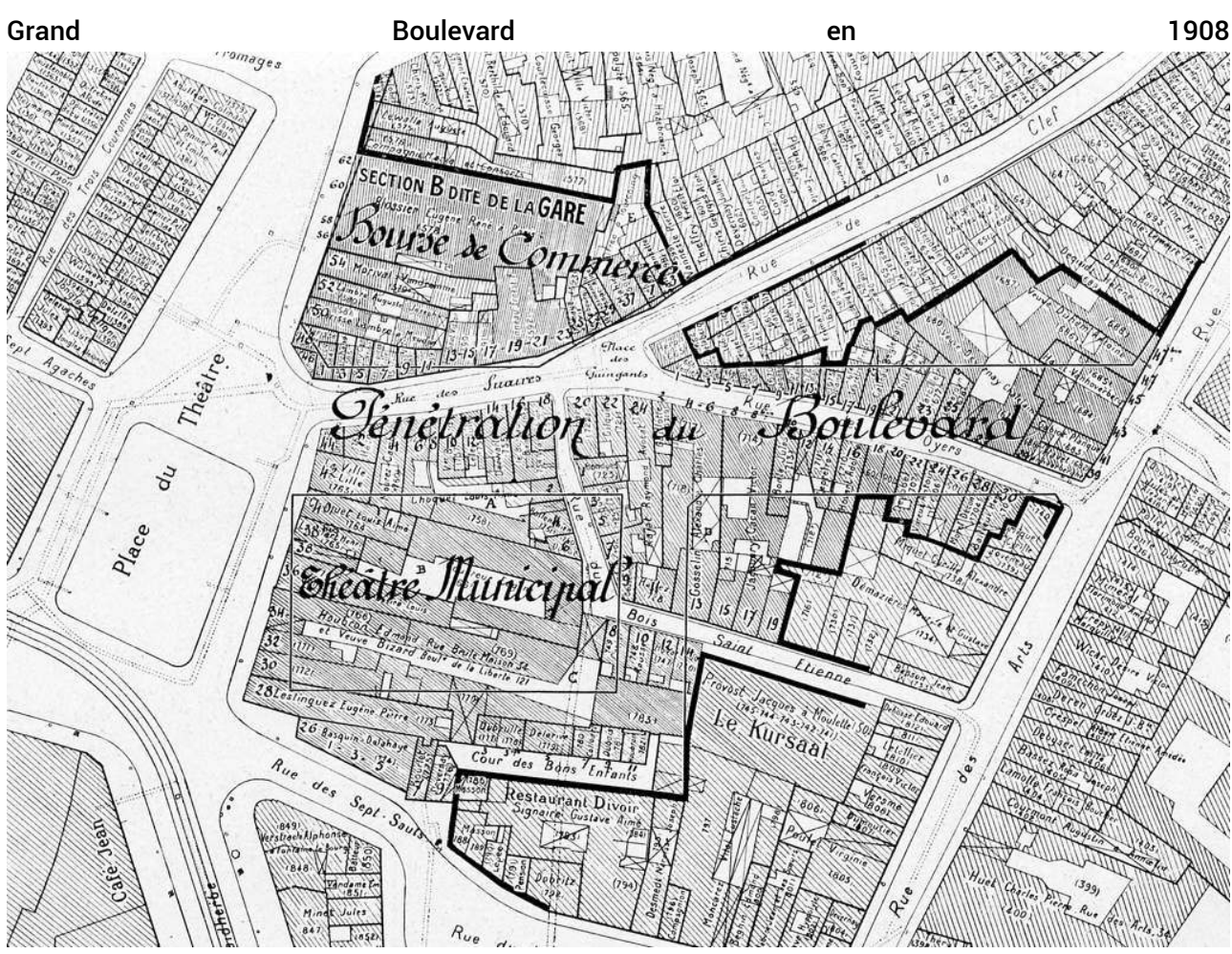

Planche gravée, Bulletin de la Commission historique du département du Nord, t. 27, 1908, p. 291.

8 La municipalité propose donc un emplacement à l'entrée de ce grand boulevard dont la construction est imminente ${ }^{18}$. La configuration future de cet espace urbain se met alors en place par le déplacement du futur théâtre à reconstruire à l'entrée de la nouvelle voie, avec une façade tournée vers la Vieille Bourse et la Grand'Place. Par concours, la construction du nouveau théâtre sera confiée à l'architecte Cordonnier en $1907^{19}$ qui choisira un style néo-gabriel répondant au programme académique d'une salle de spectacle. En 1905, la Chambre nomme en son sein une commission spéciale chargée de rechercher un local suffisamment spacieux pour permettre un regroupement de tous les services et de locaux commerciaux destinés à la location. Un comité d'études se forme parmi les négociants et les courtiers intéressés et offre sa collaboration à la Chambre. Le projet est également soutenu par l'administration préfectorale qui y voit un outil de promotion du commerce régional : «La création d'une nouvelle bourse de commerce est depuis longtemps et très instamment réclamée par les commerçants de la région et la presse s'est maintes fois fait l'écho des doléances des intéressés. Il faut reconnaître que ces doléances sont justifiées et que l'installation d'une bourse nouvelle, capable de répondre à toutes les exigences modernes, est de nature à accroître l'importance du centre commercial lillois, pour en faire un centre régional de premier ordre $\aleph^{20}$. La commission de la Chambre examine la proposition municipale le 12 janvier 1906 et charge Cordonnier d'établir des avant-projets en ce sens ${ }^{21}$. La collaboration entre l'architecte et la Chambre ne date pas du projet de nouvelle bourse, puisque dès 1897, dans le contexte de recherche d'un nouveau local, Cordonnier avait été invité à dresser les plans d'un édifice à construire rue de la chambre des comptes dans lequel la Chambre s'installa l'année suivante ${ }^{22}$. C'est également à lui que la Chambre s'adressa en 1904 pour élaborer des plans en vue du transfert des services consulaires dans le marché Saint-Nicolas ${ }^{23}$. 
Cordonnier faisait donc figure d'architecte officiel de la Chambre de commerce bien avant le projet de la bourse.

Dans la séance du 16 février 1906, nous apprenons qu'un projet réduit aurait été un temps préféré, mais le président de la Chambre précise que «[d]eux mois environ sont encore nécessaires pour que l'on puisse établir nettement des propositions et se prononcer ${ }^{24}$. À partir de cette date, les procès-verbaux de la Chambre indiquent laconiquement que « [1]a partie des procès-verbaux concernant cette question [la construction d'une nouvelle Bourse de Commerce] sera publiée séparément ${ }^{25}$, ce qui ne semble avoir jamais été le cas. Il faut désormais suivre le développement de l'histoire aux Archives départementales et dans l'Historique de la Chambre publié en 1921. Ce dernier nous apprend notamment que la municipalité s'était engagée à faire les démarches nécessaires pour une acquisition à l'amiable des immeubles à détruire pour la construction, mais que la Chambre s'inquiétait des voies et moyens selon l'expression consacrée. Malgré la proposition de la Ville, des expropriations n'étaient pas à exclure et un emprunt devenait de toute façon indispensable pour solder les travaux ${ }^{26}$. La Ville se proposait ainsi de contribuer à hauteur de $20 \%$ de la dépense totale sans pour autant dépasser la somme de 500000 frs et elle chargea M. Duvilla, déjà négociateur pour le compte du département dans les expropriations du nouveau boulevard, d'obtenir des promesses de vente amiables. En trois mois, il obtint l'accord de 16 des 21 propriétaires pour une somme de $1850000 \mathrm{frs}^{27}$. Le 16 mars 1906, les travaux de la commission spéciale et les avant-projets préparés par Cordonnier furent examinés par la Chambre (ill. 2) ${ }^{28}$.

III. 2 : L.-M. Cordonnier, « Projet B' (avec café). (2e Rez-de-chaussée). Bourse », s.d. [mars 1906 ?]

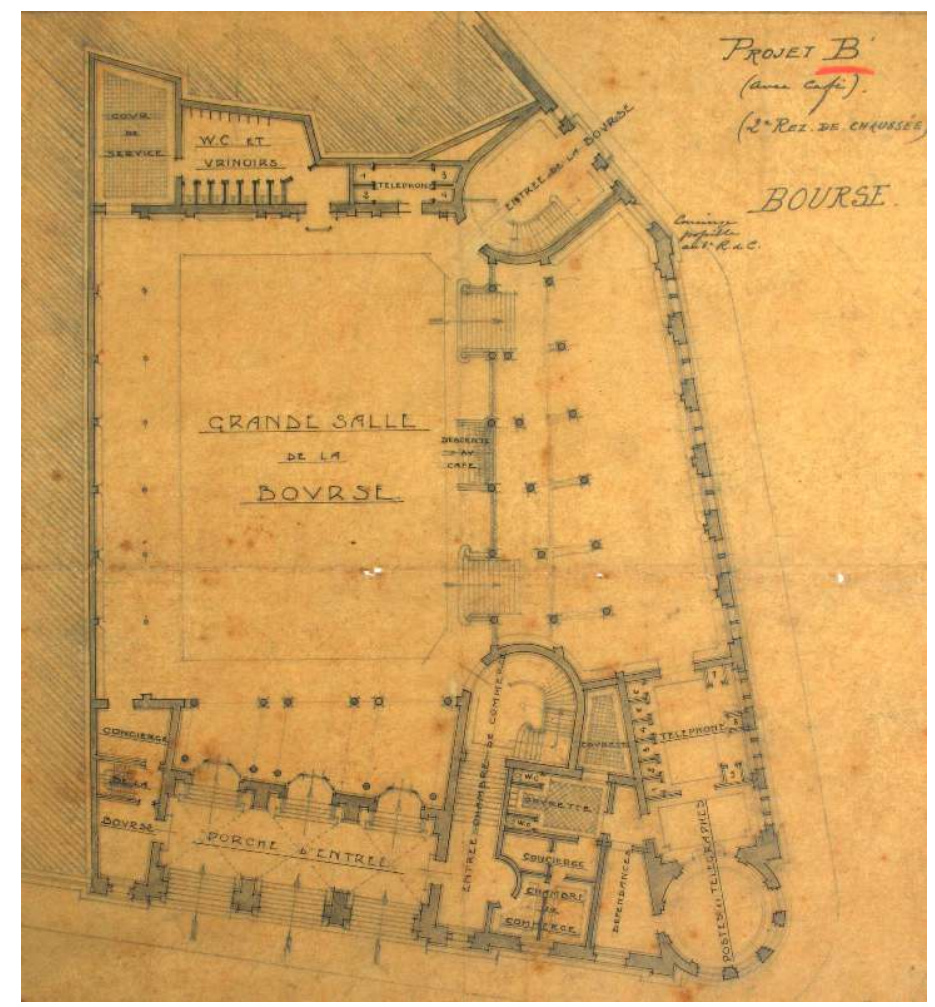

ENCRE ET CRAYON SUR CALQUE, ARCH. DÉPART. DU NORD, 136J222/7

Cl. Olivier Liardet 
Elle « adopta le principe de l'érection d'une nouvelle Bourse sur l'emplacement convenu » 29. Il s'agissait désormais pour la Ville de réaliser les acquisitions avant l'expiration des délais des promesses de vente afin de concrétiser l'amélioration du centre ville : «Tous les avantages de l'opération nous paraissent indiscutables. Nous acquérons à des prix raisonnables des terrains situés en plein cœur de la Ville. Nous amorçons l'entrée du boulevard de Roubaix-Tourcoing et commençons un assainissement que nous espérons poursuivre. Enfin, nous dotons la Ville d'un beau monument dont la nécessité s'impose ${ }^{30}$ . » Après acquisition, la municipalité devait rétrocéder les terrains à la Chambre à charge pour elle d'entreprendre seule l'édification du nouveau bâtiment. Trois jours plus tard, un plan parcellaire est dressé et le préfet signe un arrêté autorisant l'enquête publique pour l'acquisition des terrains, close le 20 avril suivant par l'avis favorable du maire. L'emplacement choisi se révéla assez incommode malgré une situation exceptionnelle au débouché du nouveau boulevard au centre de la ville. Il s'agit en effet d'un large pentagone irrégulier d'immeubles anciens possédant de nombreuses caves qui, pour certaines d'entre elles, s'étendent sous la voirie communale (ill. 3) ${ }^{31}$.

\section{3 : Émile Dubuisson, vue de l'îlot démoli pour faire place à la nouvelle Bourse, état en 1908}

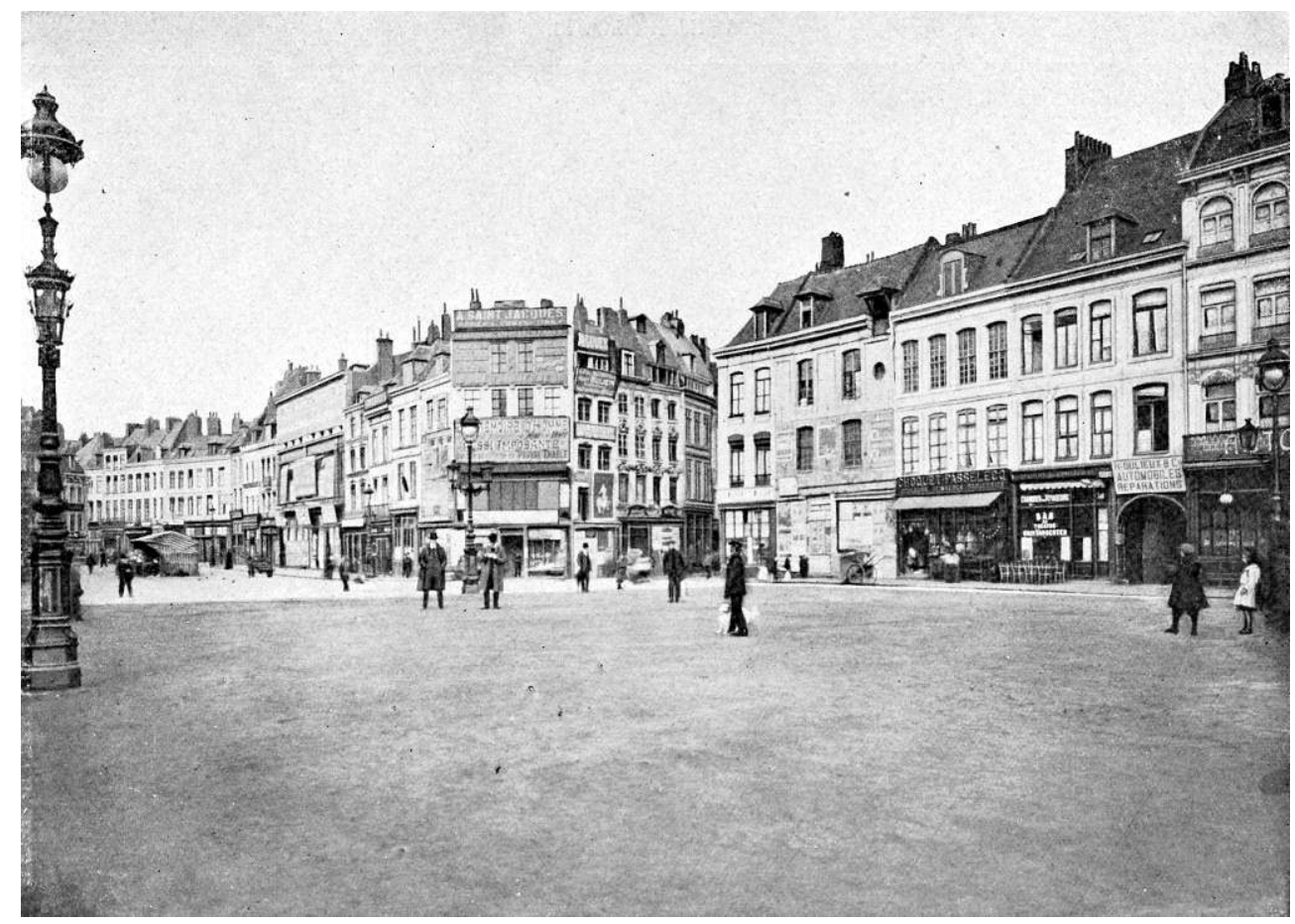

Photographie, Bulletin de la commission historique du département du Nord, t. 27, 1908, p. 294.

11 Dès lors, la construction sur un tel emplacement constitue-t-elle un véritable défi que l'architecte de la nouvelle Chambre, nommé officiellement le 4 mai 1906, va précisément s'employer de relever ${ }^{32}$.

\section{Le premier projet de Cordonnier}

12 Le fonds d'agence de l'architecte conserve de nombreuses esquisses préparatoires pour l'édifice lillois, mais il est assez difficile de déterminer leur correspondance avec les premiers projets de 1906 comme à celui adopté en 1909 (ill. 4a à f). 
III. 4a à f : L.-M. Cordonnier, esquisses préparatoires pour le projet de nouvelle Bourse montrant les variations proposées sur ce thème, s.d. [1906-1909]
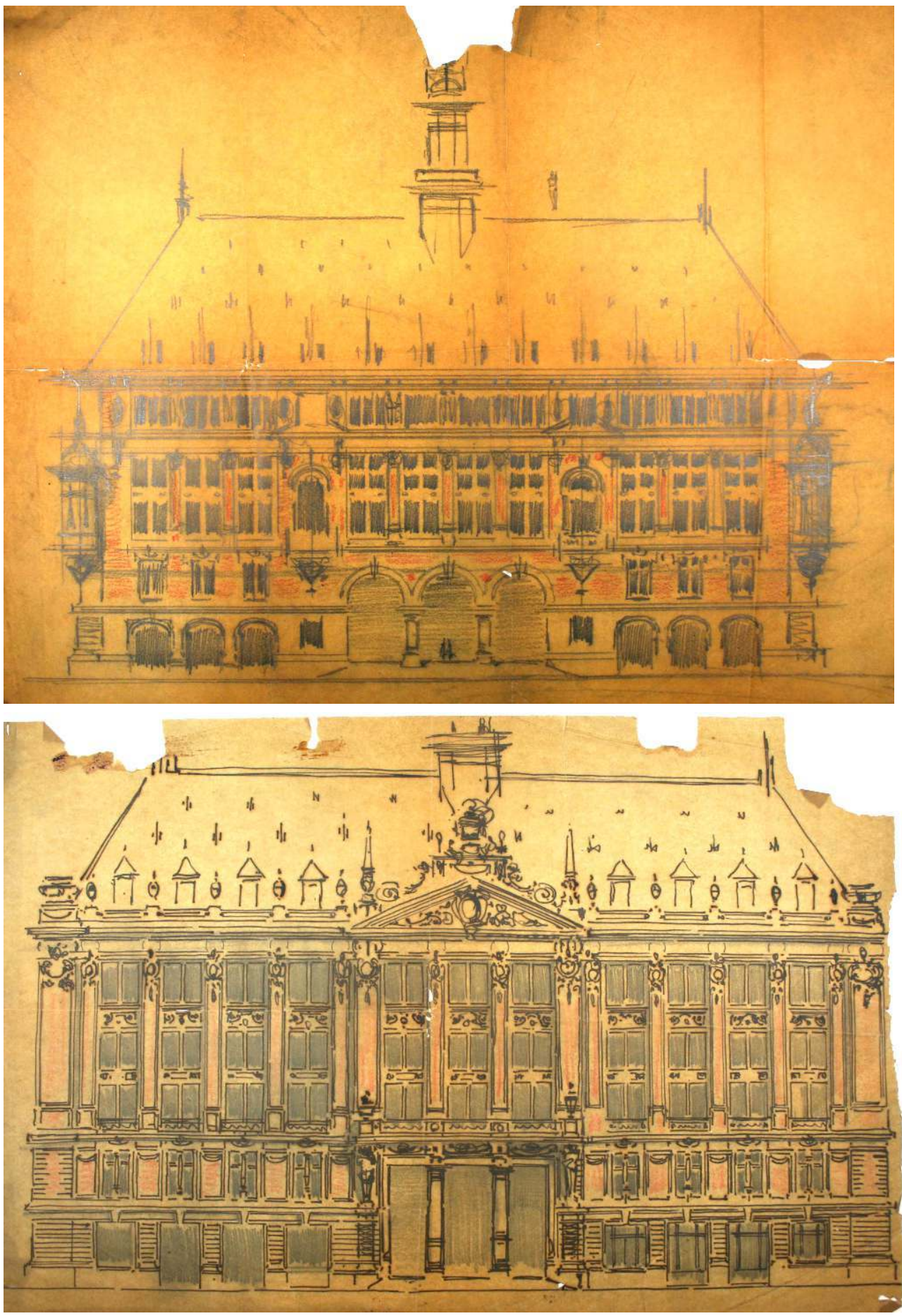

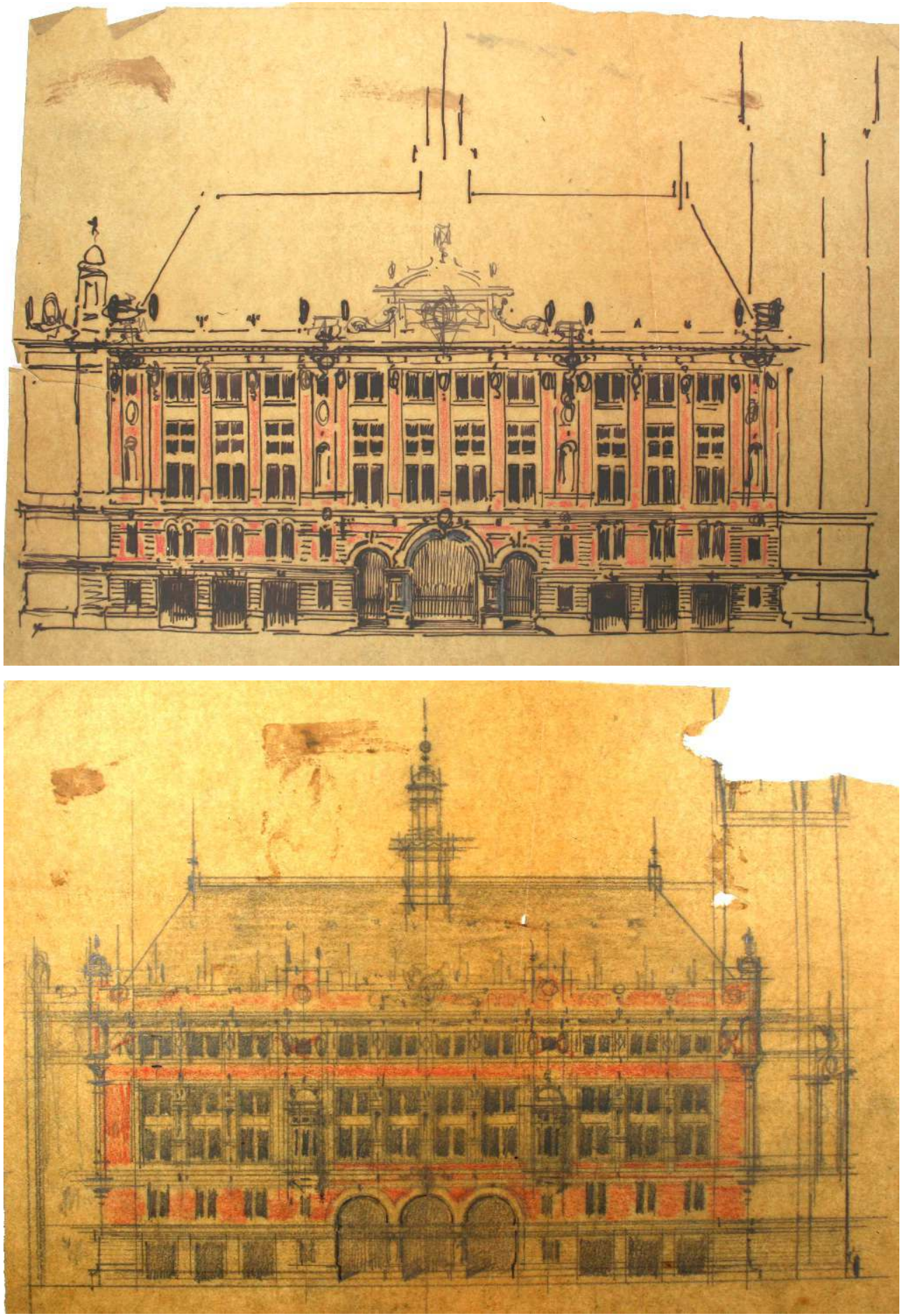

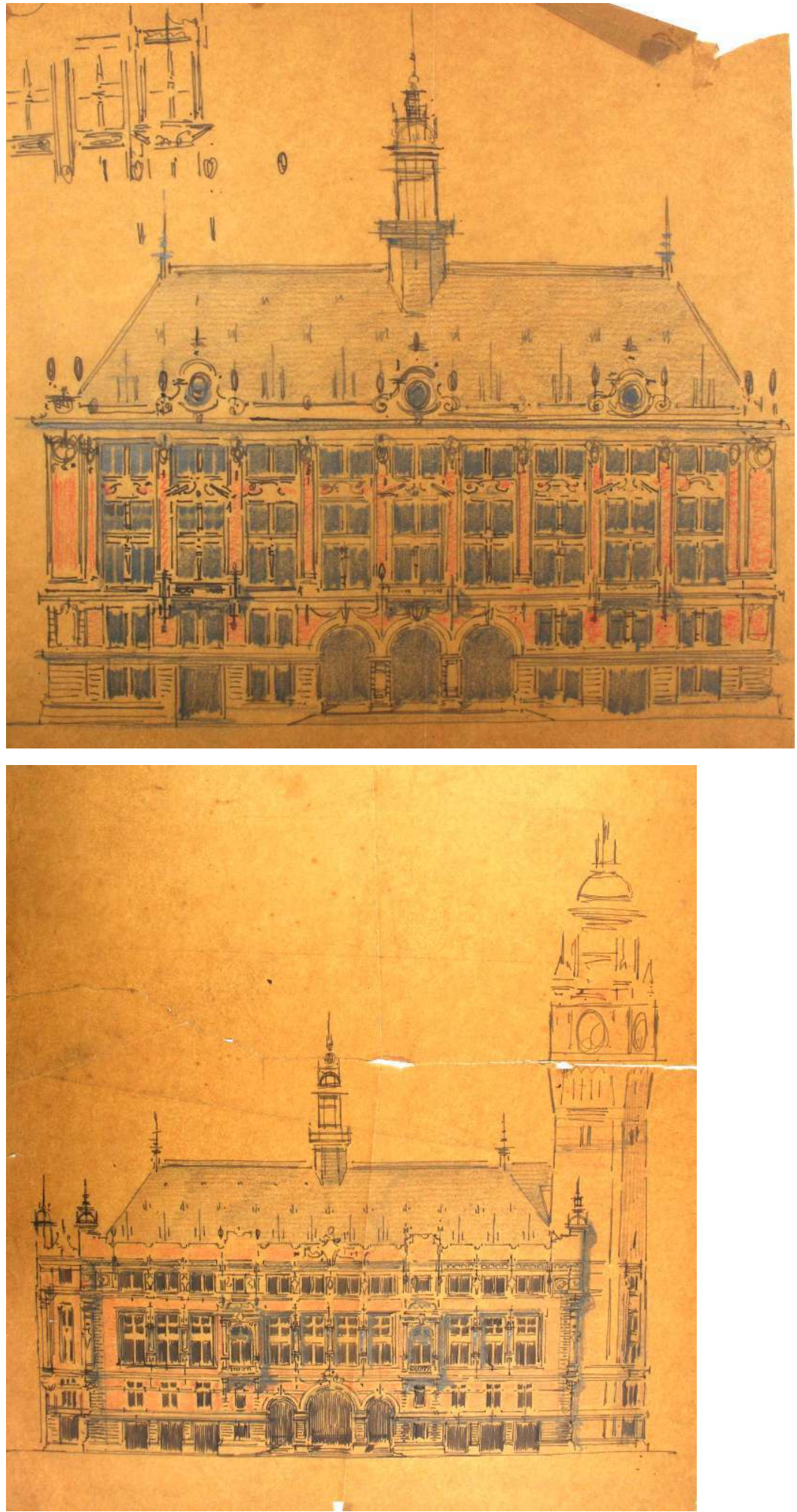

Crayons, encre et lavis sur calque, Arch. départ. du Nord, 136J222/2

\section{OLIVIER LIARDET}

13 À l'exception d'une seule élévation, aucun de ces dessins n'est daté ${ }^{33}$. Les partis déclinés présentent de nombreuses similitudes et constituent autant de variations sur le thème du grand corps de bâtiment de division ternaire couvert d'une imposante toiture d'ardoises. Les parties latérales encadrent une partie centrale bien marquée, soit par une séparation 
de type fenêtre encadrée par des colonnes et balcon ou bow-window, soit par un avantcorps surmonté d'un grand fronton. Une première série d'esquisses (la plus importante) peut être isolée en raison de l'absence du beffroi ou bien de son rajout évident en complément d'un dessin préalable. Les unes s'inspirent très fortement de la Renaissance, avec la présence de fenêtres à croisées et de tourelles en encorbellement dans les angles de la construction, les autres se rapprochent plus volontiers des modèles maniéristes ou des premières tentatives du baroque flamand. En tout état de cause, toutes ces propositions revendiquent leur allégeance aux grandes constructions publiques de la seconde moitié du XVI ${ }^{e}$ siècle à la fin du XVII ${ }^{e}$ siècle des anciens Pays-Bas, arborant des façades mêlant la brique rouge et la pierre.

Le premier projet daté de Cordonnier, produit le 13 juillet $1906^{34}$, présente déjà les grandes caractéristiques des propositions successives, c'est-à-dire une grande salle centrale distribuant l'ensemble des services répartis autour d'elle, créant de nombreux espaces tampons découlant de la forme de la parcelle mais sur un terrain réduit. Le centre de la façade s'ouvre par trois porches et devait être surmonté par un clocheton ouvragé, des tours d'angle venant fermer la composition (ill. 5).

\section{5 : L.-M. Cordonnier, « Département du Nord. Ville de Lille. Projet de Bourse de Commerce. Façade principale », Lille, le 13 juillet 1906}

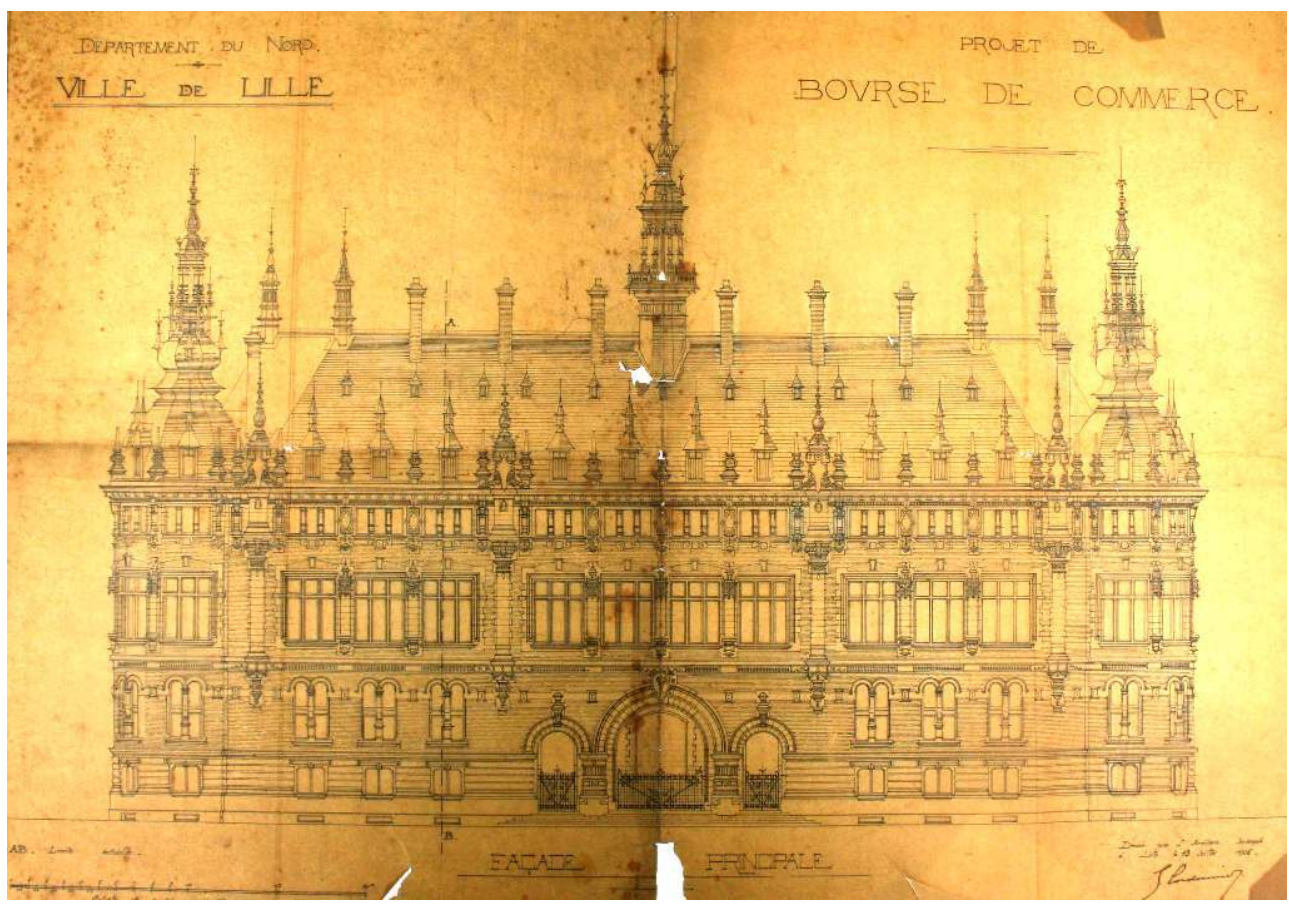

ENCRE SUR CALQUE, ARCH. DÉPART. DU NORD, 136J222/7

Cl. Olivier Liardet

15 Cordonnier opte ainsi pour une façade néo-Renaissance flamande très inspirée par les travaux des architectes belges et hollandais tels Henri Beyaert (1823-1894) ou P.-J. Cuypers (1827-1921). L'Opéra, de même que la bourse, sont invités à magnifier l'entrée du nouveau boulevard dont les travaux sont alors en cours d'exécution. Le 6 octobre suivant, le projet annexait des parcelles adjacentes rendant plus cohérentes les façades du 13 juillet avec les nouveaux plans ${ }^{35}$. Le devis s'élève à 1282625 frs, somme à laquelle s'ajoutent les 1895000 frs nécessaires à l'acquisition des terrains ${ }^{36}$. La Ville de Lille ayant 
voté 500000 frs de subvention, il s'agit donc pour la Chambre de trouver pas moins de 2700000 frs par voie d'emprunt. Le projet est soumis à l'examen du conseil général des bâtiments civils sur le rapport rédigé par Louis Sortais le 11 octobre $1906^{37}$. Mais de quel projet s'agissait-il, celui du 13 juillet ou celui du 6 octobre? Peut-être les deux projets ont-ils été présentés simultanément afin d'offrir une vision complète de l'ensemble du bâtiment. Le rapporteur ne dissimule pas son admiration pour le grand architecte septentrional: «Les façades sont prévues en pierre et briques; avec leurs campaniles, leurs clochetons et pinacles, leurs hautes toitures propres aux villes du Nord, elles donnent bien l'idée du style brillant et mouvementé habituel à l'architecte; c'est peut être un peu hôtel de ville ; mais l'ensemble est séduisant, gai et coloré ", avant de conclure très favorablement, "en résumé, les dessins qui nous sont soumis nous paraissent très achevés, bien présentés, et répondent très probablement à tous les desiderata de la chambre de commerce qui les présente comme siens. D'autre part, le talent reconnu de l'auteur par les belles constructions qu'il a édifiées ou projetées nous sont un sûr garant de l'intérêt que présentera sa nouvelle œuvre ». Une seule critique est émise par le Conseil : «Considérant que les dispositions générales du parti exprimé par les nouveaux plans sus-visés sont satisfaisantes; qu'il convient de regretter toutefois que l'architecte n'ait pas saisi l'occasion qu'il avait de composer une grande salle intérieure qui eût pu être très belle et monumentale au lieu que celle qu'il a indiquée manque d'étude et de liaison avec l'ensemble ${ }^{38}$. » Le devis est définitivement approuvé le 25 octobre suivant.

Les décrets présidentiels des 19 décembre 1906 et 17 janvier 1907 autorisent respectivement la Ville à exproprier et contracter un emprunt de 500000 frs et la Chambre à emprunter $2678125 \mathrm{frs}^{39}$. Afin de se prémunir contre des imprévus possibles, une convention entre les parties est signée le 15 février 1907 et approuvée par décret le 27 avril. Les expropriations peuvent alors commencer et le 3 mai 2007 le tribunal civil de $1^{1}$ ère instance de l'arrondissement de Lille prononce l'expropriation définitive des immeubles à l'amiable et du 21 au 27 février 1908 les cas litigieux sont réglés ${ }^{40}$. Les travaux de démolition sont adjugés le $1^{\text {er }}$ juillet 1908 et ceux-ci sont presque achevés à la fin de 1908.

\section{Le projet définitif}

Le projet présenté au conseil général des bâtiments civils était probablement destiné à donner des gages à toutes les parties de l'avancement effectif du projet, mais visiblement, il ne s'agissait pas d'une proposition réalisable en l'état. Cordonnier et deux membres de la commission de la Chambre, Edmond Faucheur et Georges Masquelier, visitent à partir de décembre 1906 les bourses de Londres et de Liverpool. En juillet 1908, Alfred Descamps, membre et futur président de la Chambre, qui prit également une part active dans l'élaboration des projets depuis 1906, les accompagne pour visiter les bourses de Brême, Hambourg et Amsterdam ${ }^{41}$. Ces édifices isolés montraient combien le terrain dévolu à la construction lilloise était incapable de recevoir un monument digne de l'institution consulaire et la commission proposa en conséquence à la Chambre l'agrandissement jusqu'à la rue de la Grande-Chaussée pour aboutir au développement présenté par la première élévation de Cordonnier. Une seconde convention intervint donc entre la Ville et la Chambre le 10 décembre 1908 afin de régler ce problème ${ }^{42}$. Lors de la transmission de la deuxième demande d'emprunt, le président de la Chambre indique le 27 janvier 1909 les raisons de ce nouveau développement : « Comme vous l'avez 
apprécié vous-même, le projet primitif de la nouvelle Bourse, dont la façade principale s'arrêtant brusquement place du Théatre, se trouvait imparfait et il apparaissait à tous nécessaire de prolonger le monument jusqu'à la rue de la Grande-Chaussée. La chambre de commerce et la Ville de Lille se sont mises d'accord pour parfaire l'œuvre entreprise : ce prolongement devant permettre, en outre, de donner plus d'ampleur aux services intérieurs et d'y ajouter le service du conseil des prud'hommes $»^{43}$. En effet, la loi du 28 mars 1907 avait augmenté considérablement le nombre des juges et des justiciables de cette instance judiciaire et la Ville devait trouver des locaux pour les accueillir convenablement. La Ville s'engage ainsi à fournir 350000 frs pour les acquisitions des nouvelles parcelles et la Chambre contracte un nouvel emprunt de $1330000 \mathrm{frs}^{44}$.

Les plans et devis des constructions complémentaires dans lesquels le beffroi apparaissait clairement désormais sont examinés en 1909 par le conseil général des bâtiments civils. Le 17 juin 1909, Alexandre Marcel relève devant le Conseil l'insuffisance des informations envoyées par Cordonnier et le Conseil réclame au ministre de l'Intérieur les premiers plans portant le timbre d'approbation du Conseil afin de comparer les deux projets ${ }^{45}$. Marcel présente son rapport le 29 juillet. Malgré d'évidentes lacunes dans le dossier (absence de plan du $1^{\text {er }}$ étage et de coupe et même d'élévation), le rapporteur ne tarit pas d'éloges : «En résumé, les projets qui nous sont soumis répondant vraisemblablement à tous les desiderata de la chambre de commerce qui les présente, le talent universellement [barré !] reconnu de l'auteur du Projet étant un sûr garant de la parfaite tenue de sa nouvelle œuvre ${ }^{46}$. " Le Conseil adopte le projet dans sa séance du 7 octobre 1909 en attirant néanmoins l'attention de l'architecte sur le «manque de concordance entre les plans et les façades qui sont restées ce qu'elles étaient dans le projet primitif ${ }^{47}$.

Les façades devaient encore évoluer considérablement et la deuxième série d'esquisses, de même que trois projets plus aboutis conservés dans le fonds de l'agence, nous permettent de suivre la pensée de l'architecte. L'ensemble s'inspire largement du XVII siècle lillois pour les façades, en particulier du Rang du Beauregard (îlot de maisons régulières), situé immédiatement en vis-à-vis dont la reconstruction et le nouvel alignement dû à Simon Vollant dataient de 1687 et qui selon Paul Parent «symbolise [...] l'union du style Louis XIV et des pratiques locales les plus invétérées ${ }^{48}$. La Bourse de Cordonnier se pose ainsi en variation sur le thème de l'architecture publique de cette construction civile, laissant les éléments décoratifs supportant les balcons et ornant les linteaux du $2^{e}$ étage et ceux du beffroi dialoguer avec les sculptures de Julien Destrez à la Vieille Bourse. Cette dernière vient en contrepoint marquer l'apogée de la "Renaissance flamande » alors que le Rang du Beauregard marque la première étape d'assimilation de l'architecture française dans le respect de la tradition locale qui entre en résonance avec la propre démarche de Cordonnier qui insuffle une rigueur toute académique à la nouvelle architecture flamande. Les trois grands projets conservés dans le fonds d'agence présentent tous la même disposition pour la façade principale en vue frontale : le beffroi à droite, avec à gauche le départ de la rue de la Grande-Chaussée et à droite celui du GrandBoulevard et la partie arrière de l'Opéra. La physionomie générale de l'édifice, le nombre et les divisions des niveaux, le décor sculpté et ses différents éléments constitutifs tels que le porche central à trois arches, le beffroi d'angle et le campanile, ne varient pas d'un projet à l'autre. Si le premier des projets présentait encore des éléments renvoyant à la Renaissance flamande, les deux autres se rattachent délibérément à cette nouvelle architecture régionale présentant notamment dans la partie d'habitation des trumeaux faisant office de pilastres sur trois niveaux. 
ce deuxième projet, l'ampleur de la construction aurait permis le développement d'activités susceptibles de rentabiliser l'opération. Une brasserie, un restaurant ainsi que des magasins divers et surtout près de 150 bureaux étaient prévus. Même si certains de ces éléments étaient déjà présents dans le premier projet comme le précise le préfet du Nord en réponse aux critiques du Conseil d'État qui voyait dans l'acquisition des nouvelles parcelles une opération purement financière, leur multiplication profitait de l'agrandissement de la façade principale et du développement de la toiture d'un niveau supplémentaire (ill. 6).

III.6 : L.-M. Cordonnier, « Ville de Lille. Projet de Bourse de Commerce. Plan du second rez-dechaussée », Lille, février 1909

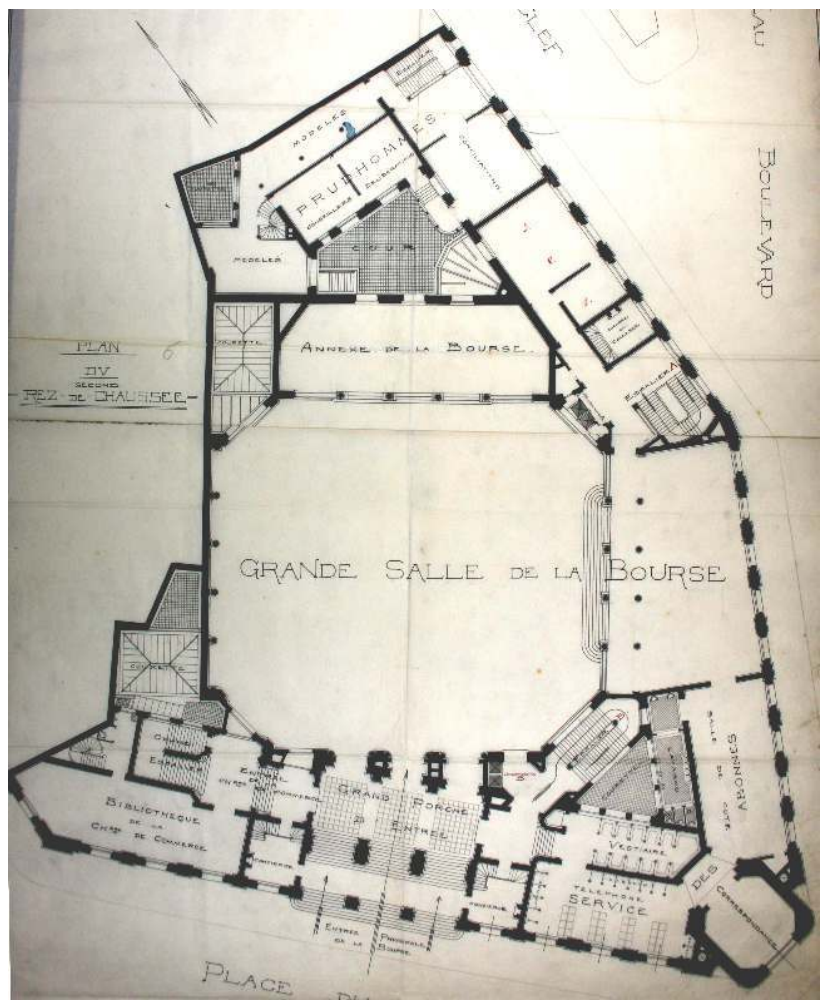

ARCH. DÉPART. DU NORD, 136J222/7

Cl. Olivier Liardet

Le sous-sol se répartissait entre les services de la Chambre (chauffage, incendie, etc), de la brasserie et d'un restaurant (caves et cuisines). Au rez-de-chaussée, deux entrées permettaient d'accéder à la grande salle de la brasserie située sous la salle des pas-perdus, tandis que la façade sur le nouveau boulevard accueillait un café-restaurant. De part et d'autre du grand perron d'accès de la façade principale s'ouvraient des guichets de la Banque de France et des Postes. L'arrière de l'édifice abritait sur deux niveaux (rez-dechaussée et entresol) le conseil des prud'hommes. C'est à l'entresol que se situait l'accès principal aux espaces de la Chambre proprement dits, en particulier la salle des pasperdus avec ses dégagements latéraux. À partir des niveaux suivants ( $1^{\mathrm{er}}$ au $5^{\mathrm{e}}$ étages), il ne s'agissait plus que d'accueillir des bureaux et quelques espaces de représentation placés en façade principale, à savoir l'escalier d'honneur, un vestibule desservant le cabinet du président, la salle de commissions et la salle des séances dont les plafonds à voussures occupent un niveau complet ( $2^{\mathrm{e}}$ étage). Avec la salle des pas-perdus, ces espaces 
de réception sont les seuls à présenter des décors sculptés ou peints et un mobilier conçu spécialement à leur destination. Les bureaux sont desservis essentiellement par deux grands escaliers, l'un situé dans l'angle de la salle des pas-perdus dans l'axe du beffroi et l'autre au début de la rue de la Clef permettant un accès séparé pour les bureaux destinés à être loués. D'après plusieurs témoignages contemporains, la combinaison de ces plans intérieurs doit beaucoup à la collaboration de l'architecte avec Alfred Descamps qui visait la rentabilisation maximale de l'espace ${ }^{49}$. Finalement, l'idée de la brasserie et du caférestaurant ne résista pas à la guerre et le bureau de poste s'installa sur la façade latérale dans un espace beaucoup plus vaste ${ }^{50}$. D'ailleurs, la Chambre utilisa immédiatement l'ensemble de son nouvel hôtel consulaire en raison du développement de ses activités de soutien à l'industrie au cours de la première guerre mondiale et de son rôle majeur dans la Reconstruction ${ }^{51}$.

Les nouveaux travaux sont déclarés d'utilité publique le 2 juillet 1909. Si la première brique est posée le 22 septembre 1909, la première pierre de la tour du nouvel édifice ne l'est que le 15 avril 1910 seulement par le président de la Chambre en présence de Cordonnier ${ }^{52}$. Quelques photographies du chantier nous permettent d'en saisir les grandes étapes. Alors que le soubassement et les niveaux bas de l'édifice sont déjà réalisés, une grande structure métallique supportant une grue prend place à l'intérieur de l'édifice à l'emplacement de la future salle des pas perdus afin de hisser les blocs et poutrelles métalliques de construction dans les futurs étages et les combles (ill. 7).

\section{7 : Vue du chantier de la nouvelle Bourse arrivé au niveau des toitures de l'Opéra avant 1914}

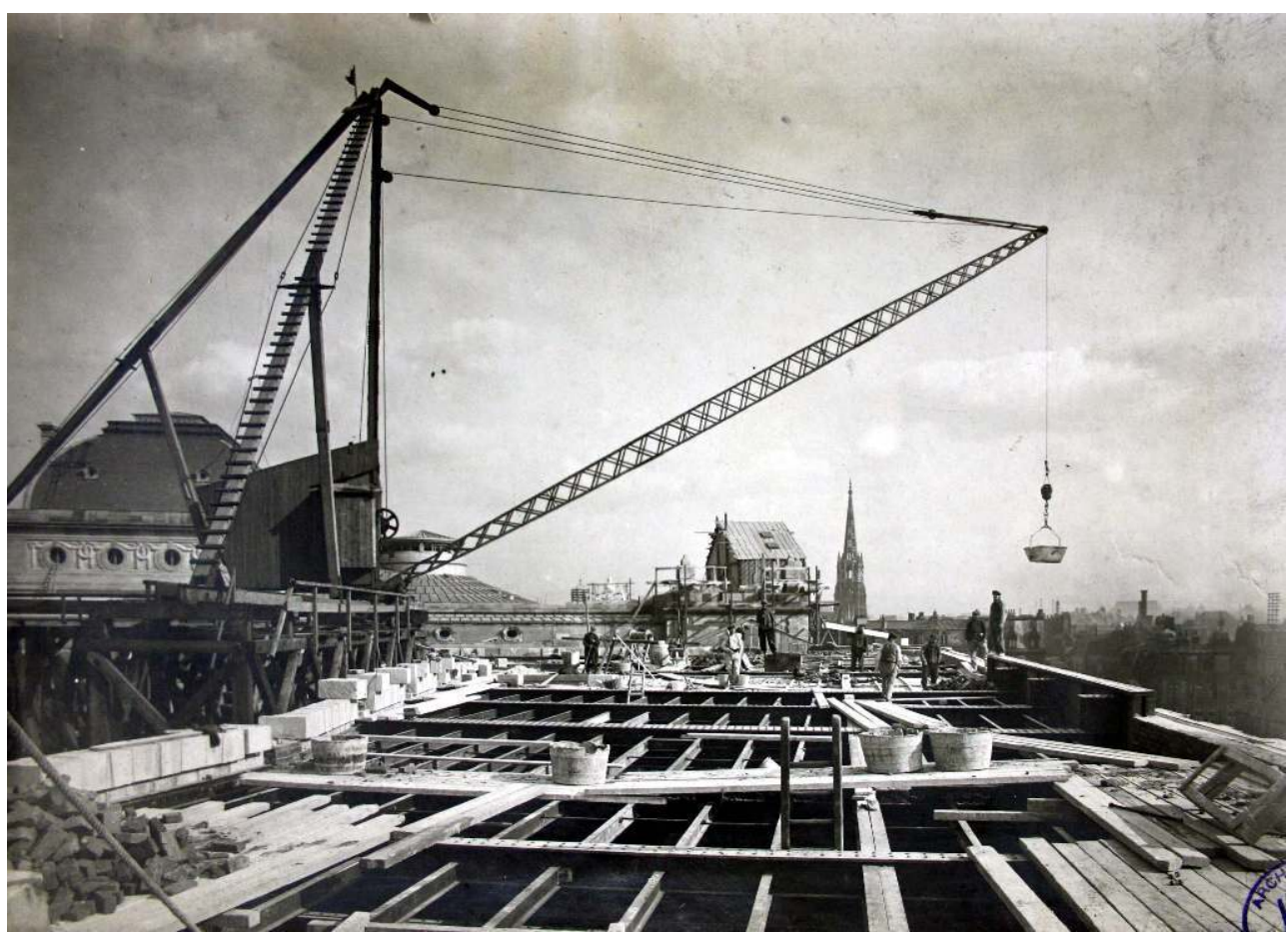

PHOTOgRAPHIE CONSERVÉE DANS LE FONDS CORDONNIER, ARCH. DÉPART. DU NORD, 136J65

Cl. Olivier Liardet

Lorsque la Grande Guerre éclate, le gros-œuvre est à peu près terminé, le conseil des prud'hommes est déjà installé dans ses bureaux tandis que l'aménagement intérieur devait être achevé pour la fin de l'année 1914. L'occupation allemande interrompt le 
chantier au profit de l'achèvement de l'Opéra qui sera inauguré par l'occupant. En 1915, les locaux sont mis à la disposition du comité d'alimentation hispano-américaine tandis que l'espace de la brasserie sert d'entrepôt aux décors de l'Opéra. En avril 1916, l'hôtel de ville est détruit par un incendie et les édiles émettent l'idée d'utiliser la nouvelle Bourse pour installer les services municipaux : « Le Maire trouvait [...] que le monument, avec ses dispositions intérieures, se prêterait facilement à une adaptation en hôtel de ville. Il faisait appel aux sentiments de bons lillois qui animent les membres de la Chambre pour accueillir favorablement sa demande et venir en aide à la Ville dans la situation difficile où l'avait placée le désastreux incendie $"^{53}$. Le rapport de Georges Masquelier confirme aux membres du conseil de la Chambre l'impossibilité d'une cession eu égard aux sacrifices consentis pour cette construction et aux bienfaits attendus pour l'industrie régionale ${ }^{54}$. Un dernier emprunt de 5000000 frs, autorisé par décret du 29 mai 1920, est encore nécessaire pour achever la construction ${ }^{55}$. L'interruption du chantier pendant la guerre, de même que l'inflation consécutive à celle-ci et les réclamations des entrepreneurs à partir de 1919, devaient engendrer une sérieuse dérive financière.

\section{Un manifeste néo-régionaliste}

Même si des exemples d'édifices d'inspiration flamande voient le jour dès le Second Empire, succédant aux expériences "régionalistes » néo-médiévales et néo-gothiques en particulier ${ }^{56}$, Louis-Marie Cordonnier est considéré comme le premier architecte néorégionaliste septentrional ou tout au moins celui par lequel le néo-flamand acquiert ses véritables lettres de noblesse ${ }^{57}$. Sa première œuvre d'importance, l'hôtel de ville de Looslez-Lille, conçu à partir de 1883 témoigne déjà d'une approche originale de la tradition architecturale flamande dans une interprétation néo-Renaissance remise au goût du jour à la même époque par les architectes belges et hollandais ${ }^{58}$. Alors que la Belgique apparaît comme le véritable conservatoire du passé architectural flamand, la France tentera de «nationaliser » le régionalisme septentrional en l'associant de fait au mouvement initié depuis une génération visant à redécouvrir l'architecture de la Renaissance française. Mais dans le cas présent, la Renaissance flamande est comprise comme une période plus large allant pratiquement jusqu'à l'annexion française qui apporte à Lille les formules d'un classicisme jusqu'alors inconnu localement. Ainsi Louis-Charles Boileau découvre-t-il les maisons anciennes du Vieux-Lille conçues «dans ce même esprit de la Renaissance flamande, Henri IV et Louis XIII, un peu chargé de bossages, de cartouches, de gaines et de frontons circulaires interrompus, contournés de toutes sortes de façon, mais en somme ingénieux. Les architectes modernes y peuvent trouver nombre d'idées originales ${ }^{59}$. À travers une construction archéologique savante, Cordonnier développe une architecture d'inspiration régionaliste dont les sources se veulent purement françaises, en accord avec les besoins de la vie moderne, réunissant la tradition décorative flamande et la rigueur architecturale française issue de la tradition académique : «Considérant avec juste raison que le climat, les habitudes et les matériaux de la région du Nord ne sont pas les mêmes qu'à Paris et ailleurs, il a voulu donner une couleur locale à ses conceptions en faisant revivre les traditions de l'architecture des Flandres, tout en tenant compte des idées modernes. Il a démontré ainsi par ses œuvres que l'on pouvait produire des effets nouveaux avec des éléments anciens et en donnant une physionomie spéciale à ses constructions il a prouvé qu'en province l'on pouvait ériger des constructions intéressantes et artistiques très différentes de celles de Paris ${ }^{60}$. " 

devenant un élément obligé dans les constructions d'hôtels de ville, à l'image de ceux construits par Cordonnier à La Madeleine près de Lille ou encore à Dunkerque. La Bourse de Lille revendique à son tour cette filiation "municipale » et le rapporteur au conseil général des bâtiments civils ne manquera pas de le souligner (ill. 7). Face à la Vieille Bourse et au Rang du Beauregard, le beffroi de 76 mètres de hauteur devient le symbole même de la puissance consulaire et par là-même la construction la plus haute de Lille. D'ailleurs, la démolition du Rang du Beauregard et des îlots parallèles a été envisagée un temps par le plan d'urbanisme "Lille Cœur des Flandres » conçu par Jacques Gréber (1882-1962) et Louis-Stanislas Cordonnier (1884-1960), fils de Louis-Marie, lauréat du concours de 1920 pour le plan d'aménagement, d'embellissement et d'extension de la ville exigé par la loi Cornudet du 14 mars $1919^{61}$. Dans ce grand projet urbain, la nouvelle Bourse devait tenir un rôle éminent puisque la démolition du Rang du Beauregard dégageait complètement sa façade principale avec le beffroi depuis la Grand'Place par la création d'une place ornée d'une fontaine monumentale lointain rappel de l'ancienne fontaine aux changes démolie pour la construction de la Vieille Bourse. L'institution consulaire se plaçait ainsi comme une concurrente de l'institution municipale par cette position privilégiée. On comprend dans ses conditions que la municipalité ait chargé un autre architecte de réaliser un plan d'urbanisme moins destructeur pour la ville ancienne et moins coûteux. Émile Dubuisson (1873-1947) s'attacha surtout au développement d'un pôle municipal autour du nouvel hôtel de ville au sein du quartier Saint-Sauveur « régénéré ». L'édifice fut construit à partir de 1924 et l'architecte l'adossa à un beffroi en béton armé de plus de 100 mètres de hauteur qui détrônait la tour consulaire quelques années seulement après son achèvement ${ }^{62}$.

La nouvelle Bourse de Lille fut unanimement saluée par les contemporains tout autant que le regroupement des services consulaires : «On comprend que la modeste galerie de la vieille Bourse ne lui suffisait plus et qu'elle l'abandonne sans regrets. Les agents de change et le public seront désormais plus à l'aise et pourront, en outre, profiter de tous les avantages que leur offrent les installations de la belle œuvre de Louis Cordonnier. Aussi le transfert de la Bourse des valeurs dans le nouvel édifice a-t-il été bien accueilli par tous et notre population se félicite hautement de voir désormais, réunies dans un même local, les différentes « Bourses » de notre ville ${ }^{63}$. " Si les services s'installèrent dans la nouvelle Bourse dès avant la fin de l'année 1920, il n'y eut pourtant pas d'inauguration officielle immédiate malgré les efforts déployés par les membres de la Chambre pour intéresser les autorités de tutelle à leur installation, sans succès : «La visite de M. Isaac, ministre du commerce, a marqué cette prise de possession, et un banquet intime [et non pas officiel], dans la somptueuse salle des fêtes de la chambre de commerce, en a souligné l'importance $\aleph^{64}$. Le président de la Chambre profita cependant de cette occasion pour prononcer un discours de légitimation de l'action consulaire et de rappeler les raisons de la nouvelle construction: "Devant l'insuffisance de l'ancienne Bourse, joyau de notre cité, construite en 1651 pour les marchands, mais devenue beaucoup trop exiguë en raison du développement industriel et commercial de notre région, notre Compagnie s'est vue obligée en 1906 de décider la construction d'une nouvelle Bourse. Elle a voulu que le monument projeté devienne le centre des affaires, que les assises commerciales puissent s'y tenir régulièrement, que les étrangers y trouvent toutes les facilités pour leurs transactions et qu'un grand nombre de bureaux particuliers soient mis à la disposition du 
commerce et de l'industrie. Elle a voulu aussi que ce monument du travail, tout en restant sobre, pratique et moderne, fût digne de notre Ville et elle en a confié l'érection à son éminent concitoyen, Louis Cordonnier, membre de l'Institut. Je suis heureux de rendre ici un public hommage à son talent et de lui dire avec tous ses admirateurs combien son œuvre est appréciée. Le but poursuivi est atteint et si les travaux, malheureusement interrompus par la Guerre et si difficilement poussés depuis la cessation des hostilités, n'ont pas encore permis d'organiser tous les services, le monument est d'ores et déjà devenu une véritable ruche, dont l'emblème se trouve sur ses murs et dans laquelle la chambre de commerce avait à juste titre sa place marquée ${ }^{65}$. "

Cordonnier, grand gagnant de l'aventure lilloise, récoltait une fois encore tous les suffrages. Sa nouvelle Bourse de Lille, véritable plaidoyer en faveur de l'industrie et du commerce régionaux, devenait le témoin privilégié d'un régionalisme triomphant et de l'« âme flamande ", un monument par lequel la Renaissance septentrionale s'unissait à la Renaissance Française dans une même ambition : «Lorsqu'on la compare à la bourse de commerce de Paris, l'avantage est à la nôtre, et la Bourse parisienne paraît triste, en dépit d'une colonnade circulaire qui n'arrive pas à lui enlever son aspect morose. Tandis qu'à Lille, les colonnes de porphyre qui séparent la rotonde du porche ou des salles annexes, les revêtements de marbre du sol, les belles mosaïques de quelques parties planes des murailles et qui sont dues au peintre d'Espouy, et surtout la lumière dorée qui tombe de la coupole, donnent à cette immense salle de la Bourse quelque chose d'unique, une somptuosité sereine et resplendissante ${ }^{66}$.»

\section{NOTES}

1. 1. Le fonds Cordonnier est conservé aux Archives départementales du Nord sous la cote $136 \mathrm{~J}$. Les principales références bibliographiques sont les suivantes: Aude Cordonnier, Louis-Marie Cordonnier: ses hôtels de ville avant 1914, mémoire de maîtrise d'histoire, université Lille 3, Villeneuve d'Ascq, 1980, 3 vol., 353 p.; Théâtre et architecture: Louis-Marie Cordonnier (1854-1940) architecte, cat. exp., Lille, Opéra-École d'architecture, 1985, 100 p. ; Odile Lesaffre, « Louis-Marie Cordonnier et l'architecture du Nord de la France », Les Pays-Bas français, t. 23, 1998, p. 45-64 ; AKL ,t. 21, 1999, p.195-196.

2. 2. Sur l'histoire des institutions consulaires et de la Chambre de Lille, voir Historique de la chambre de commerce de Lille 1714-1918, Lille, 1921, 146 p. (abrégé désormais en Historique) et JeanPierre Debourse (dir.), C comme "Centenaires». Histoire d'entreprises centenaires de la région NordPas de Calais, Lille, 1992, p. 47-49. Les archives de la chambre de commerce ne sont pas classées et demeurent inaccessibles, mais les procès-verbaux ont été imprimés depuis 1832. Les Archives départementales $\mathrm{du}$ Nord conservent un certain nombre de documents dans la série $\mathrm{O}$ concernant l'ancienne et la nouvelle bourse (0357/1381 à 1401, 1809-1914) ainsi que dans le fonds Cordonnier $(136 \mathrm{~J} 45,65,124,220$ à 226 , soit près de 1000 dessins, quelques photographies du chantier et de la maquette ainsi que des copies de devis).

3. 3. L. Quarré-Reybourdon, "La "Bourse" de Lille", Réunion des sociétés des beaux-arts des départements, Paris, 1892, p. 312-345; Paul Parent, L'Architecture civile à Lille au XVII ${ }^{e}$ siècle, Lille, 1925, p. 115-137 ; P. Piétresson de Saint-Aubin, «La Bourse de Lille », Congrès archéologique de 
France, CXX ${ }^{\mathrm{e}}$ session, 1962, Flandres, Paris, 1962, p. 193-197 ; Jacqueline Dion, « Reconnaître et comprendre les sculptures de la Vieille Bourse de Lille », Revue du Nord, t. LXXI, n² 281, avril-juin 1989, p. 363-375 ; Christiane Lesage, «La Vieille Bourse de Lille. Nouveaux regards. Nouvelles approches ", Commission historique du Nord. Bulletin, t. XLIX, 1996-1997, Lille, 1999, p. 97-126.

4. 4. Arch. départ. du Nord, $0357 / 1381$ et 1383 , projet d'horloge à la base de la tour par l'architecte B.-J. Dewarlez-Lepers en 1808-1809 et réfection partielle de la décoration intérieure en 1840 .

5. 5. Arch. départ. du Nord, 0357/1384, travaux adjugés le 29 mars 1842 à Dubrulle, maîtremenuisier à Lille, et réglés le 22 décembre 1842 .

6. 6. Arch. départ. du Nord, $0357 / 1386$ et 1387.

7. 7. Statue aujourd'hui déposée au palais des beaux-arts de Lille.

8. 8. Les derniers éléments de la décoration seront installés de 1886 à 1896.

9. 9. A.C.C.L., t. 7, 1863-68, p. 197-198.

10. 10. À l'exception de quelques années notamment pendant les travaux d'extension de l'hôtel de ville dans les locaux du Palais Rihour sur les plans de Benvignat. De 1845 à 1847, la chambre de commerce loue un local à la chambre des notaires au 11 rue des Fossés, puis de 1847 à 1850, elle tient ses séances dans la salle des délibérations du tribunal de commerce au palais de justice. À partir de 1868, la chambre s'installe dans un local aménagé spécialement par la compagnie des chemins de fer du Nord au-dessus de la salle des pas-perdus de la gare de Lille nouvellement agrandie. Marie-Josèphe Lussien-Maisonneuve, «Du débarcadère à la gare : l'exemple de Lille. Mises au point et réflexions ", Revue du Nord, t. LXII, n 245, avril-juin 1980, p. 459-473. Voir A.C.C.L., t. 7, 1863-68, p. 147-149, séance du 19 juillet 1864.

11. 11. A.C.C.L., t. 29,1894 , p. 325 , séance du 21 septembre.

12. 12. A.C.C.L., t. 29,1894 , p. 345 , séance du 5 octobre.

13. 13. A.C.C.L., t. 31,1896, p. 458-59, séance du 28 décembre, p. 462-64, séance du 22 décembre.

14. 14. Ancien élève du peintre Léon Bonnat à Paris.

15. 15. A.C.C.L., t. 31,1896 , p. 643 , séance du 13 novembre.

16. 16. A.C.C.L., t. 40, 1905, p. 388-89, séance du 15 septembre, p. 390 et 397-399, séance du 22 septembre, p. 455-457, séance du 20 octobre.

17. 17. Bernard Ménager, "Les affrontements politiques et sociaux (1851-1914)», dans Louis Trénard et Yves-Marie Hilaire (dir.), Histoire de Lille, t. 4, « Du XIX ${ }^{\mathrm{e}}$ siècle au seuil du XXI ${ }^{\mathrm{e}}$ siècle », Paris, 1999, p. 79-80.

18. 18. A.C.C.L., t. 40, 1905 , p. 456 , séance du 20 octobre.

19. 19. En plus des ouvrages sur Cordonnier, voir Jeanne Bossuyt, L'Opéra de Lille, Itinéraire du patrimoine, $n^{\circ} 148$, Paris, 1997, non paginé [16 p.].

20. 20. Arch. départ. du Nord, 0357/1391, minute de lettre du préfet du Nord au ministre de l'intérieur, 19 juillet 1906.

21. 21. Historique, p. 119.

22. 22. A.C.C.L., t. 32,1897 , p. 214 , séance du 16 avril.

23. 23. A.C.C.L., t. 39, 1904, p. 40, séance du 29 janvier.

24. 24. A.C.C.L., t. 41, 1906, p. 74, séance du 16 février.

25. 25. A.C.C.L., t. 41, 1906, p. 260, séance du 18 mai.

26. 26. La nouvelle loi de 1898 donnant le statut d'établissement public aux Chambres leur offre la possibilité de contracter des emprunts notamment pour la construction d'un édifice destiné à abriter leurs services.

27. 27. Arch. départ. du Nord, 0357/1392, extrait du registre des délibérations du conseil municipal, séance du 23 mars 1906.

28. 28. Deux plans désignés comme "Projet B' » font peut-être partie de cette phase d'avantprojets (Arch. départ. du Nord $136 \mathrm{~J}$ 222/7).

29. 29. Historique, p. 119-120. 
30. 30. Arch. départ. du Nord, 0357/1392, extrait du registre des délibérations du conseil municipal, séance du 23 mars 1906.

31. 31. Émile Dubuisson, Vues du quartier démoli pour la construction de la bourse de commerce et du nouveau théâtre à Lille, Lille, 1908, 29 p. (extrait du Bulletin de la commission historique du département du Nord, t. 27, p. 289-315).

32. 32. Historique, p. 120.

33. 33. Arch. départ. du Nord, $136 \mathrm{~J} 222 / 1$ et 2 , esquisses au lavis sur calque.

34. 34. Arch. départ. du Nord, 136J222/7, cinq plans, une élévation, et $0357 / 1397$, deux plans (tirages).

35. 35. Arch. départ. du Nord, 136J222/7, plan général du rez-de-chaussée et quatre plans partiels de la partie supplémentaire.

36. 36. Arch. départ. du Nord, 136J124, plusieurs devis partiels et récapitulatifs.

37. 37. Arch. nat., $\mathrm{F}^{21} 6520$, dossier complet comportant les différents rapports et minutes des procès-verbaux de 1906 et 1909.

38. 38. Arch. nat., $\mathrm{F}^{21}$ 6520, avis du conseil général des bâtiments civils, 11 octobre 1906 (à la suite du rapport de Sortais). Remarque faite par Julien Guadet et Victor Laloux.

39. 39. Arch. départ. du Nord, 03571391 et 0357/1394.

40. 40. Arch. départ. du Nord, $0357 / 1397$, notice explicative du service des expropriations, 4 février 1909. Les acquisitions et expropriations coûtèrent finalement 1772500 frs ; Historique, p. 121-122.

41. 41. Historique, p. 122.

42. 42. Arch. départ. du Nord, $0357 / 1397$, copie de la convention.

43. 43. Arch. départ. du Nord, $0357 / 1400$, lettre du président de la Chambre au préfet du Nord, 27 janvier 1909.

44. 44. Il en faudra encore deux autres pour parvenir à l'achèvement du bâtiment : 3500000 frs (décret du 12 avril 1913), 5000000 frs (décret du 29 mai 1920).

45. 45. Arch. nat., $\mathrm{F}^{21}$ 6520, minute du procès-verbal du 17 juin 1909.

46. 46. Arch. nat., $\mathrm{F}^{21}$ 6520, rapport d'A. Marcel du 24 juillet 1909.

47. 47. Arch. nat., $\mathrm{F}^{21}$ 6520, minute du procès-verbal du 7 octobre 1909.

48. 48. Paul Parent, L'Architecture civile à Lille au XVII ${ }^{e}$ siècle, Lille, 1925, p. 184.

49. 49. A.C.C.L., t. 47, 1912, p. 88, séance du 9 février ; Historique, p. 126.

50. 50. Dès 1911, la Chambre se met en rapport avec l'administration des postes pour le financement de ce nouveau bureau ; voir A.C.C.L., t. 47, 1912, p. 28, séance du 19 janvier, p. 88-89, séance du 9 février.

51. 51. En 1950, une aile fut ajoutée au début de la rue Grande-Chaussée : ces aménagements vinrent compléter l'offre de bureaux; la salle des pas-perdus s'agrandit d'une annexe à gauche à l'intérieur d'un pâté de maisons et reçut une peinture représentant l'inauguration du port de Lille en 1951. Une salle de réunion fut aménagée à l'emplacement prévu pour la brasserie en 1975.

52. 52. Historique, p.125.

53. 53. Edmond Faucheur, Lille et la chambre de commerce pendant la Guerre 1914-1918, Lille, 1922, regroupant les A.C.C.L. des années concernées, t. 51, 1916, p. 19-20.

54. 54. Ibid., p. 22-29.

55. 55. A.C.C.L., t. 55, 1920, p. 299-300, annexe C à la séance du 4 juin.

56. 56. Voir Frédéric Vienne, «Charles Leroy (1816-1879), l'homme qui a planté le drapeau du gothique dans le département du Nord », dans F. Vienne (dir.), Notre-Dame de la Treille. Du rêve à la réalité. Histoire de la cathédrale de Lille, Marseille, 2002, p. 140-167.

57. 57. Sur le mouvement néo-régionaliste, voir Jean-Claude Vigato, L'Architecture régionaliste. France 1890-1950, Paris, 1994, 390 p. ; pour la Flandre, se reporter en particulier à Benoît Mihail, 
Une Flandre à la française. L'identité régionale à l'épreuve du modèle républicain, Saintes (Belgique), $2006,422 \mathrm{p}$.

58. 58. François Loyer, "Ornement et caractère ", dans Le Siècle de l'éclectisme. Lille 1830-1930, Paris-Bruxelles, 1979, p. 65-104, voir en particulier p. 90 et suivantes. Voir également Anne Van Loo, « Belgique : contre la « francisation » de la culture », dans François Loyer et Bernard Toulier (dir.), Le Régionalisme, architecture et identité, Paris, 2001, p. 126-133.

59. 59. L.-C. Boileau, dans L'Architecture et la construction dans le Nord, t. VI, 1897, p. 69-70 (cité par B. Mihail).

60. 60. «Fête lilloise en l'honneur de M. Louis Cordonnier », dans L'Architecture et la Construction dans le Nord, t. XVI, 1906, p. 94 (cité par B. Mihail).

61. 61. Voir Claude Fouret et Michel Vangheluwe, L'Aventure des villes. Les villes du Nord de la France de 1850 à 1950, Lille, Arch. départ. du Nord, 1997, p.99-107 et Marie-Josèphe Lussien-Maisonneuve, dans Histoire de Lille, t. 4, Paris, 1999, p. 205-209. Le projet est publié dans Le Grand Hebdomadaire illustré de la région Nord de la France, $3^{\mathrm{e}}$ année, n 6, 6 février 1921, p. 41-43 et L'Architecture, vol. XXXIV, 1921, p. 3-6, pl. XIII-XIV.

62. 62. Jean-Étienne Grislain, "L'hôtel de ville de Lille un palais pour le peuple », Les Cahiers de la recherche architecturale, $\mathrm{n}^{\circ} 24 / 25,1^{\mathrm{er}}$ et $2^{\mathrm{e}}$ trimestres 1989, p. 55-64.

63. 63. Le Grand Hebdomadaire [...], $2^{\mathrm{e}}$ année, $\mathrm{n}^{\circ} 51,19$ décembre 1920, p. 402

64. 64. Le Grand Hebdomadaire [...], $2^{\mathrm{e}}$ année, $\mathrm{n}^{\circ}$ 50, 12 décembre 1920, p. 394.

65. 65. A.C.C.L., t. 55, 1920, p. 690-691, allocution du président de la Chambre au dîner offert au ministre du commerce et de l'Industrie le 4 décembre. La venue dans le Nord du président de la République, Alexandre Millerand, le 16 mai 1921, fut l'occasion de lui présenter l'édifice pendant une trentaine de minutes, mais le cabinet présidentiel tint bon sur le fait que cette visite ne devait pas être considérée comme une inauguration officielle (voir les pourparlers et discours dans A.C.C.L., t. 56, 1921, p. 10-11, 253, 273, 317, 336-343).

66. 66. Le Grand Hebdomadaire [...], $2^{\mathrm{e}}$ année, $\mathrm{n}^{\circ}$ 50, 12 décembre 1920, p. 395.

\section{RÉSUMÉS}

Au cours du XIX ${ }^{e}$ siècle, le développement de l'institution consulaire à Lille amène la conception d'un édifice spécifique destiné à abriter des services toujours plus nombreux. La construction de cette nouvelle bourse de commerce intervient après une longue errance et de multiples projets d'installation. C'est à l'architecte septentrional Louis-Marie Cordonnier (1854-1940), déjà célèbre et reconnu, que les travaux sont confiés en 1906. Ils sont l'occasion de mettre à profit les expériences urbaines que sont l'ouverture du boulevard reliant Lille à Roubaix et Tourcoing et la reconstruction de l'opéra (confiée elle aussi à Cordonnier). Ce dernier réalise pour la chambre de commerce un véritable monument à la gloire de l'industrie régionale et de l'architecture de tradition flamande, devenant, lors de son inauguration en 1920, un signal urbain puissant dans une ville en quête de renouvellement.

The development of the consular institution in Lille throughout the nineteenth century led to the project of facilities specially designed to house expanding services. The erection of this new Stock Exchange followed a long wandering time and several housing projects. The already known and acknowledged Northern architect Louis-Marie Cordonnier (1854-1840) was entrusted with the construction work in 1906. He was thus given the opportunity to turn to good account urban 
experimentations such as the laying of the boulevard from Lille to Roubaix and Tourcoing and the reconstruction of the opera house (also appointed to Cordonnier). The latter achieved a true monument dedicated to the glory of local industry and Flemish architectural tradition for the Chamber of Commerce: at the opening time in 1920 it became a striking urban signal in a city in pursuit of renewal.

Im Laufe des 19.Jahrhunderts machte die Entwicklung der konsularischen Institution in Lille die Gestaltung eines spezifischen Gebäudes für die Unterbringung der stetig wachsenden Verwaltungsdienste notwendig. Der Bau dieser neuen Handelsbörse kam nach mehreren wechselhaften Errichtungsprojekten zustande. Damit beauftragt wurde 1906 der aus Nordfrankreich stammende Architekt Louis-Marie Cordonnier, (1854-1940) der schon berühmt und anerkannt war. Aus diesem Anlass wurden urbane Experimente versucht, so zum Beispiel die Eröffnung einer Verbindungsstraße zwischen Lille, Roubaix und Tourcoing, und der Wiederaufbau der Oper, auch von Cordonnier gebaut. Für die Handelskammer errichtete der Architekt ein Gebäude zu wahrhaftem Ruhm und Ehre der regionalen Industrie und der Architektur flämischer Tradition. 1920 eingeweiht stand es sofort als starkes Zeichen für den Erneuerungswillen der Stadt.

\section{AUTEUR}

\section{OLIVIER LIARDET}

Olivier Liardet est chargé d'études documentaires à la conservation régionale des monuments historiques du Nord-Pas-de-Calais depuis septembre 2004 et, à ce titre, chargé de la protection au titre des monuments historiques. Ses publications portent en particulier sur les travaux de l'architecte Charles-Auguste Questel (1807-1888) : «La restauration de la chapelle royale de Versailles 1873-1878. Charles-Auguste Questel et les pratiques restauratrices à la fin du XIX ${ }^{\mathrm{e}}$ siècle », Versalia, n 6, 2003 ; «La reconstruction de la Galerie dorée de la Banque de France par Charles Questel (1865-1875)», dans Place des Victoires. Histoire, architecture, société, Paris, 2003 ; «L'église Saint-Paul de Nîmes. Charles-Auguste Questel et le processus créatif en architecte. 1835-1849 ", dans L'Architecture religieuse au XIX ${ }^{e}$ siècle entre éclectisme et rationalisme, Paris, PUPS, 2006. Ses intérêts portent sur l'architecture publique du XIX ${ }^{\mathrm{e}}$ siècle : « La cour des Comptes ", dans La Place Vendôme. Art, pouvoir et fortune, Paris, 2002 ; « Le ministère de la guerre. Des bureaux de la Guerre à l'îlot Saint-Germain ", Livraisons d'histoire de l'architecture (n 8, 2004). Il s'est également intéressé à la création architecturale à Nîmes et dans le Gard dans la première moitié du XIX siècle : «Charles-Étienne Durand (1762-1840) à Nîmes ou le rêve de la ville néo-classique à l'aube du XIXe siècle ", dans Autour de la ville de Napoléon. Colloque de la Roche-sur-Yon, Rennes, PUR, 2006. Depuis 1999, il participe à l'élaboration de notices biographiques d'architectes et d'ingénieurs français du XIX ${ }^{\mathrm{e}}$ siècle pour l'Allgemeines Künstlerlexikon. Adresse électronique : olivier.liardet@culture.gouv.fr 